\title{
In silico analysis of highly conserved cytotoxic T-cell epitopes in the structural proteins of African swine fever virus
}

\author{
Leana Rich De Mesa Herrera(i) and Elizabeth Paulino Bisa (iD) \\ Department of Physical Sciences, College of Science, Polytechnic University of the Philippines, Manila, Philippines. \\ Corresponding author: Leana Rich De Mesa Herrera, e-mail: Irmherrera@pup.edu.ph \\ Co-author: EPB: epbisa@pup.edu.ph \\ Received: 07-06-2021, Accepted: 01-09-2021, Published online: 09-10-2021
}

doi: www.doi.org/10.14202/vetworld.2021.2625-2633 How to cite this article: Herrera LRM, Bisa EP (2021) In silico analysis of highly conserved cytotoxic T-cell epitopes in the structural proteins of African swine fever virus, Veterinary World, 14(10): 2625-2633.

\begin{abstract}
Background and Aim: African swine fever (ASF) is a viral disease of pigs caused by ASF virus (ASFV). High mortality and the lack of available treatments have severely impacted the swine industry resulting in huge global economic losses. In response to the dire necessity for vaccines, this study aims to identify highly conserved cytotoxic T-cell epitopes in ASFV structural proteins pp220, pp62, p72, p30, and CD2v through immunoinformatics approach.

Materials and Methods: The amino acid sequences of the structural proteins were retrieved from the National Center for Biotechnology Information protein database. The sequences were evaluated in CD-HIT Suite wherein resulting representative sequences were aligned in Clustal Omega. Highly conserved sequences were identified in the Protein Variability Server which were used as reference sequences for the cytotoxic T-cell epitope mapping. Epitopes were predicted using the tools in Immune Epitope Database. Peptides which bind to the swine major histocompatibility complex with $\mathrm{IC}_{50}$ binding scores $>500 \mathrm{nM}$ were filtered out. Epitopes which are classified to be potentially toxic and cross-reactive with the swine proteome sequences were all excluded from the study. The epitopes were docked with the swine leukocyte antigen- 1 *0401 (SLA$1 * 0401$ ) wherein the binding affinity, the binding energy, and the root-mean-square deviation (RMSD) per residue of epitope-SLA complexes formed were determined and compared with the influenza epitope as positive control.
\end{abstract}

Results: A total of 112 highly conserved fragments with Shannon variability index $\leq 0.1$ were identified. These include $66,12,26,6$, and 2 highly conserved fragments from ASFV proteins pp220, pp62, p72, p30, and CD2v, respectively. From these reference sequences, 35 nonameric peptides were selected for the list of candidate cytotoxic T-cell epitopes. These include 26 epitopes for pp220, 7 for pp62, 6 for p72, and one each for $\mathrm{p} 30$ and CD2v. Bioinformatics analysis classified the peptides as non-toxic. Further evaluations of epitopes showed that these are less likely to cross-react with the domestic swine proteome sequences. This study identified candidate epitopes from pp220 (IADAINQEF, FLNKSTQAY, QIYKTLLEY, and SLYPTQFDY), and pp62 (GTDLYQSAM, FINSTDFLY, and STDFLYTAI) which can bind to at least two widely distributed SLAs in pig populations. The immunogenicity of candidate peptides RSNPGSFYW, DFDPLVTFY, AIPSVSIPF, and VVFHAGSLY was validated by the acceptable binding affinities, binding energies, and RMSD of the peptide-SLA complexes formed. Results were also comparable with the crystal structure of an SLA-epitope complex in the database.

Conclusion: This is the first study to identify highly conserved cytotoxic T-cell epitopes in the structural proteins of ASFV. Overall, the results of in silico evaluations showed that the identified highly conserved cytotoxic T-cell epitopes may be used as part of future vaccine formulations against ASFV infection in domesticated pigs. Nonetheless, these findings require in vitro and in vivo validation before application.

Keywords: African swine fever virus, cytotoxic T-cell epitopes, immunoinformatics, in silico.

\section{Introduction}

African swine fever (ASF) is a viral disease of pigs with mortality approaching $100 \%$ [1]. It was first identified in Kenya in the 1920s; then, it had spread to Europe in the middle of the past century. In 1990s, ASF was eradicated from the most affected regions (except Sardinia and sub-Saharan countries) due to the implementation of biosafety regulations. But in 2007, ASF

Copyright: Herrera and Bisa. Open Access. This article is distributed under the terms of the Creative Commons Attribution 4.0 International License (http://creativecommons.org/licenses/ by/4.0/), which permits unrestricted use, distribution, and reproduction in any medium, provided you give appropriate credit to the original author(s) and the source, provide a link to the Creative Commons license, and indicate if changes were made. The Creative Commons Public Domain Dedication waiver (http:// creativecommons.org/publicdomain/zero/1.0/) applies to the data made available in this article, unless otherwise stated. rapidly spread out again from Africa. At present, ASF has been identified in Africa, Europe, America, and Asia [2-4]. ASF is caused by the ASF virus (ASFV) which can spread between soft ticks (Ornithodoros erraticus) and wild pigs through feeding, or less frequently through direct transmission between wild pigs. In domesticated pigs, ASFV can be readily transmitted resulting to lethal hemorrhagic fever. The widespread of ASF has been a great challenge for swine breeding. Although it does not pose a risk to human health, ASF has resulted to huge economic losses around the world [5]. ASFV, the only species of the genus Asfivirus within the family Asfarviridae, is an icosahedral enveloped virus with double-stranded DNA as its genetic material. The viral particle consists of an inner core coated by a thick protein core shell enclosing its genetic material. 
The subsequent layers are the lipid envelope surrounding the protein core shell, and the viral capsid which forms the outermost cover of an intracellular virion. Virions budding out of the host cell carry a portion of the host plasma membrane forming an extra layer of envelope [6]. The length of its genome differs from one isolate to another (170-194 kbp) with 151-167 open reading frames [7]. ASFV encodes around 50 structural proteins with significant roles in genome replication and viral packaging. Vital structural proteins include pp220, pp62, p72, p30, and CD2v. These major components of viral particles are important in viral attachment, entry, replication, and processing [8]. Both pp220 and pp62 are polyprotein precursors proteolytically cleaved to mature virion proteins. Structural polyprotein pp220 is encoded by gene $C P 2475 \mathrm{~L}$ which product is cleaved and post-translationally processed to $\mathrm{p} 150, \mathrm{p} 37, \mathrm{p} 14$, and $\mathrm{p} 34$. Structural protein pp62, a relatively shorter polypeptide, is encoded by gene CP530R. Similar to pp220, this polyprotein is proteolytically cleaved into mature p35 and p15 virion proteins. The post-translational products of pp220 and pp62 play roles in viral particle assembly and form the major component of the viral core shell $[9,10]$. Findings suggest that the expression of the major capsid protein p72 is a requirement for the processing of pp220 and pp62 in ASFV [11]. Antigenic structural protein $\mathrm{p} 72$ is encoded by gene B646L (VP72) which serves as a major protein component in viral capsids and functions in the formation of ASFV capsids [12]. Another crucial structural protein in ASFV is p30. It is encoded by $C P 204 \mathrm{~L}$ gene and is most abundantly expressed during the early phase of infection. It has crucial functions in viral entry and is known to be one of the most antigenic ASFV proteins $[13,14]$. Studies showed that recombinant p30 can be an efficient ASFV antibody detector both in oral fluid and serum samples [15]. ASFV gene EP402R encodes the structural glycoprotein $\mathrm{CD} 2 \mathrm{v}$ which consists of transmembrane region, extracellular domain ( $\mathrm{N}$-terminal), and cytosolic domain (C-terminal). CD2v is associated to immune response modulation and lymphocyte function impairment which enhance the virulence of ASFV in domestic swine [16]. In addition, it has been shown to be directly involved in viral hemadsorption resulting in increased spread of the virus [17].

Due to the significant roles of pp220, pp62, p72, $\mathrm{p} 30$, and $\mathrm{CD} 2 \mathrm{v}$ in ASFV replication and assembly, disporting key properties including immunogenicity, abundant expression, and virulence mechanism, these structural proteins can be suitable immunotherapeutic targets in designing vaccines and treatments against ASFV. At present, there are no anti-viral agents and vaccines available against ASFV [5]. The significant economic diminution brought about by the ASFV infection in swine populations around the world warrants accelerated vaccine development.

In the pursuit of this compelling demand, the researchers utilized immunioinformatics approach to provide potential immunotherapeutic agents against
ASFV. Immunoinformatics tools and databases can aid in hastening and reducing the cost required to identify immunogenic proteins and peptides for vaccine designs $[18,19]$. Identification of epitopes through a computational approach is an advantageous and prototypical step in the process of vaccine development before any further in vitro and in vivo evaluations are conducted.

Thus, this study aims to identify cytotoxic T-cell epitopes from the conserved amino acid sequences of pp220, pp62, p72, p30, and CD2v in ASFV. The use of highly conserved sequences in vaccine development is one of the most important factors that must be considered to resolve immune epitope evasion in the rapidly mutating viral antigens.

\section{Materials and Methods}

\section{Ethical approval}

Ethical approval is not necessary for this type of study.

\section{Study period and location}

The study was conducted from April to June 2021. The study was conducted at the Polytechnic University of the Philippines.

\section{Retrieval and identification of highly conserved sequences}

ASFV protein sequences of pp220, pp62, p72, $\mathrm{p} 30$, and CD2v were retrieved from the National Center for Biotechnology Information protein database on April 17, 2021. Search results were filtered using 24752476, 530-534, 645-646, 203-204, and 360-404 for the sequence length of pp220, pp62, p72, p30, and CD2v, respectively. The lists of representative sequences for each antigen were obtained using CD-HIT Suite (http://weizhongli-lab.org/cdhit_suite/cgi-bin/index. cgi?cmd=cd-hit) with 1.00 as sequence-identity cutoff. Unique representative sequences for each protein were aligned in Clustal Omega (https://www.ebi.ac.uk/ Tools/msa/clustalo/) to generate multiple sequence alignment. To identify highly conserved sequences for each protein, Shannon variability threshold $\mathrm{H} \leq 0.1$ was used in the protein variability server (PVS) tool (http:// imed.med.ucm.es/PVS/). The first sequence in the alignment was used as a reference and the varying residues were masked. Fragments with $\geq 9$ residues were kept for cytotoxic T-cell epitope identification.

\section{Cytotoxic T-cell epitope mapping}

Cytotoxic T-cell epitopes which can bind to widely distributed swine leukocyte antigens (SLA) SLA- ${ }^{*} 0101$, SLA- $1 * 0401$, and SLA- $1 * 0801$ in swine population [20] were mapped in the Immune Epitope Database. NetMHCcons was utilized to integrate three well-known methods: NetMHC, NetMHCpan, and PickPocket to produce more reliable results [21]. All binders with TAP and proteasome scores below zero were excluded from the study. Epitopes with major histocompatibility complex (MHC) $\mathrm{IC}_{50}<500 \mathrm{nmol} /$ $\mathrm{dm}^{3}$ are classified as good binders [22]; thus, these were further evaluated for cross-reactivity and toxicity. 
Protein-protein BLAST (BLASTp) was utilized to identify and exclude epitopes with a significant match to swine protein sequences. Sequence match with e-values $<1.0 \mathrm{e}-30$ can be cross-reactive in some allergic individuals [23]. Although this result was found in humans, it was adopted in this work to provide a more precise cutoff reference in the identification of potentially cross-reactive peptides. Epitopes were queried against the toxin peptide database in ToxinPred tool (https://webs.iiitd. edu.in/raghava/toxinpred/multi_submit.php) to identify potentially toxic peptides that can cause damage to cells through the default SVM Method [24]. Peptides which overlap with cleavage and glycosylation sites in each protein sequence were excluded from the study. All remaining peptides were included as candidate cytotoxic T-cell epitopes.

\section{SLA-epitope docking and molecular dynamics}

The crystal structure of SLA-1*0401 bound to influenza epitope (PDB ID: 3QQ3) was retrieved from the Research Collaboratory for Structural Bioinformatics Protein Data Bank (RCSB PDB). The PDB file was cleaned to obtain the isolated structure of SLA-1*0401 for subsequent docking procedures. For each structural antigen, epitope with the highest $\mathrm{IC}_{50}$ binding affinity for SLA-1*0401 was docked with the cleaned PDB structure of SLA-1*0401 in GalaxyPepDock server (http://galaxy.seoklab.org/cgibin/submit.cgi?type=PEPDOCK). The resulting top score structures of SLA-epitope complex were further refined in the GalaxyRefineComplex server (http://galaxy.seoklab.org/cgi-bin/submit.cgi?type=COMPLEX). Refined SLA-epitope structures were viewed using the iCn3D tool (https://www.ncbi.nlm.nih.gov/Structure/ icn3d/full.html). The dissociation constant $\left(\mathrm{K}_{\mathrm{d}}\right)$ and the binding energy $\left(\Delta \mathrm{G}_{\text {bind }}\right)$ of each SLA-epitope complex were calculated in PRODIGY (https://bianca.science. uu.nl/prodigy/) at $300 \mathrm{~K}$. This webserver tool uses non-interface surface properties and intermolecular contacts for predictive models [25]. Estimated $\mathrm{K}_{d}$ and $\Delta \mathrm{G}_{\text {bind }}$ values for SLA- ${ }^{*} 0401$-bound epitopes were compared with that of the influenza epitope bound to SLA-1*0401. To determine the stability of SLAepitope complex formation, molecular dynamic simulation was performed in MDWeb server (http://mmb. irbbarcelona.org/MDWeb/) to obtain the root-meansquare deviation (RMSD) plot per residue. Molecular dynamics parameters include $\mathrm{C}$-alpha Brownian dynamics in $100 \mathrm{ps}$ time, $0.01 \mathrm{ps}$ time change, $3.8 \AA$ distance between alpha carbon atoms, 10 step output frequency, and $167.36 \mathrm{~kJ} / \mathrm{mol} \AA^{2}$ force constant. This process employs the GROMACS MD setup with solvation using force-field Amber-99sb [26].

\section{Results}

Highly conserved sequences in the ASFV structural proteins

Herein, the reference sequences identified for the ASFV structural proteins pp220, pp62, p72, p30, and $\mathrm{CD} 2 \mathrm{v}$ have Shannon variability index $\leq 0.1$. Sequences of pp220, pp62, p72, p30, and CD2v yielded 66, 12, 26,6 , and 2 highly conserved fragments, respectively. Table-1 shows the positions of highly conserved fragments identified for each antigen. Conserved sequences in pp220, pp62, and p72 are widely distributed as indicated by the presence of longer fragments adjacent to each other within the full stretch of their amino acid sequences. Antigens pp220, pp62, and p72 contain highly conserved fragments with lengths ranging from 9 to 102,11 to 139 , and 9 to 51 , respectively. All these highly conserved fragments were used as reference sequences for cytotoxic T-cell mapping.

\section{Highly conserved cytotoxic T-cell epitopes in the structural proteins of ASFV}

All resulting cytotoxic T-cell epitopes mapped using the reference sequences were filtered by excluding potentially toxic and cross-reactive peptides. Table-2 shows the list of candidate cytotoxic T-cell epitopes in pp220, pp62, p72, p30, and CD2v indicating their corresponding SLA binders, sequence location, proteasome score, TAP score, and MHC $\mathrm{IC}_{50}$. A total of 35 highly conserved epitopes from the five structural proteins of ASFV were identified. These include 26 peptides from pp220, 7 from pp62, 6 from $\mathrm{p} 72$, and one each from $\mathrm{p} 30$ and CD2v. Analysis revealed that seven of these epitopes (four in pp220 and three in pp62) can bind to at least two widely distributed SLAs. In this study, all candidate cytotoxic T-cell epitopes have $\mathrm{MHC} \mathrm{IC}_{50} \leq 500 \mathrm{nmol} / \mathrm{dm}^{3}$ with positive TAP and proteasome scores.

\section{Validation of identified cytotoxic T-cell epitopes}

The PDB structure of SLA-1*0401 was utilized for the validation procedures in this study because the crystal structures of the other two SLAs (0101 and 0801) are not available in the RCSB protein data bank. Thus, for every antigen, the epitope with the lowest binding affinity (with the highest $\mathrm{IC}_{50}$ ) to SLA$1 * 0401$ was employed for the docking procedures. The candidate epitope YQYNTPIYY in CD2v binds to SLA-1*0801; thus, it was not included in the docking procedures. Peptides docked to SLA- $1 * 0401$ include pp220 RSNPGSFYW (P1) with MHC IC 30 392.5, pp62 DFDPLVTFY (P2) with $\mathrm{MHC}_{\mathrm{IC}} 30 \mathrm{324.8}$, p72 AIPSVSIPF (P3) with MHC $\mathrm{IC}_{50} 384.1$, and p30 VVFHAGSLY (P4) with MHC IC ${ }_{50} 132.3$. The influenza epitope NSDTVGWSW bound to SLA-1*0401 was used as a control to serve as a supporting reference for positive binding. Figure- 1 shows the 3D structures of complexes formed for P1, P2, P3, and P4 and influenza epitopes (yellow), with the swine MHC I (magenta and blue) binding groove.

To evaluate if the binding of epitopes to SLA$1 * 0401$ is favorable, the dissociation constant $\left(\mathrm{K}_{\mathrm{d}}\right)$ and the binding energy $\left(\Delta \mathrm{G}_{\text {bind }}\right)$ of each SLA-epitope complex formed were calculated. Table-3 shows that the binding of candidate epitopes RSNPGSFYW (P1), DFDPLVTFY (P2), AIPSVSIPF (P3), and VVFHAGSLY (P4) with SLA- $1 * 0401$ resulted in 
Table 1: Highly conserved sequences in ASFV structural proteins.

\begin{tabular}{|c|c|c|c|}
\hline ASFV antigen & Start & End & Sequence \\
\hline \multirow[t]{54}{*}{ pp220 } & 1 & 13 & M G N R G S S T S S R P P \\
\hline & 21 & 68 & 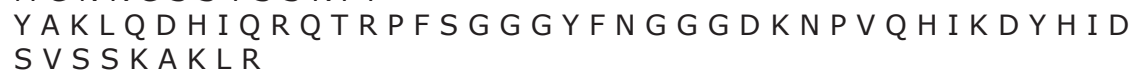 \\
\hline & 85 & 119 & D TKQP I E D I LKD I K K Q LPDPR A G S TFVKN A E K Q E T \\
\hline & 121 & 144 & CKMIADA I N Q E F D L G Q D KLIDTT \\
\hline & 146 & 186 & GAASICRQIVLYINSLTHGLRAEYLDVHGS IENTLENIKLL \\
\hline & 188 & 232 & $\begin{array}{l}\text { D A I K Q L H } \\
\text { ERMVTEVTKAAPNEEVINAVTMIEAVYRRLLNEONLOI }\end{array}$ \\
\hline & 234 & 253 & I L T N F I D N I LTPTQKELDKL \\
\hline & 264 & 291 & LNDTN S V L G T K N F G KVLSYTLCN L G I A A \\
\hline & 293 & 302 & V A N K I N K A L Q \\
\hline & 304 & 312 & V G L K VEQYL \\
\hline & 319 & 361 & EFDKELDLKRFSGLVSAENIAEFEKAVNLLRQTFNERHKILEN \\
\hline & 380 & 412 & EAQRLDRKHILMEFLNKSTQAYNDFLENVKKI G \\
\hline & 414 & 426 & KLVKE I A L T P N I T \\
\hline & 428 & 451 & LRDALSR I N D M G T I A L D LSLI G F Y \\
\hline & 453 & 464 & N A A A R E E RETFL \\
\hline & 481 & 491 & D P N F K N L Y D C \\
\hline & 493 & 511 & R L L Q I I D F Y T D I V Q K K Y G G \\
\hline & 520 & 620 & $\begin{array}{l}\text { VGGAALTVEELGLSKAARSQVDLNQAINTFMYYYYVAQIY } \\
\text { SNLTHNKQEQSYEENYATILGAIAGRLMQLDTEKNARI } \\
\text { NSPAVDLARGHVGPNPGGAQE }\end{array}$ \\
\hline & 628 & 729 & 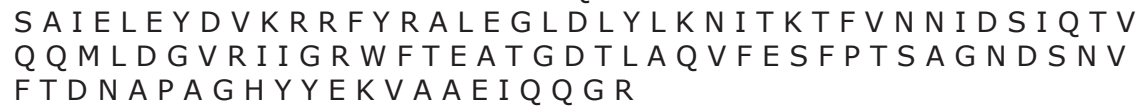 \\
\hline & 731 & 780 & $\begin{array}{l}\text { V G T LRPVRASQAKN IRDLIGRSLSNFQALKN I IN AFAR I G } \\
\text { D M L G GELRQ }\end{array}$ \\
\hline & 782 & 796 & VPMSPLQIYKTLLEY \\
\hline & 798 & 811 & Q HS A L S V G L K N L N Q \\
\hline & 830 & 844 & QRVYLSTVRVNDALS \\
\hline & 853 & 906 & $\begin{array}{l}\text { FFTFMLKSMAAKIFIVLGIYDMFERPEPVYKLIPTRMILGG } \\
\text { ADELEPEVIPEAA }\end{array}$ \\
\hline & 908 & 927 & LYFRLPRLAEFYQKLFSFRD \\
\hline & 929 & 947 & N V Q I S M LPELE G IF S G I R \\
\hline & 949 & 990 & $\begin{array}{l}\text { I F M R P I E L I I G D Y SETEIR Q L I K E I N V I Y Q H F N LEYGEQE } \\
\text { A }\end{array}$ \\
\hline & 992 & 1060 & $\begin{array}{l}\text { KKALIHFVNEINRRFGVITRTEWEKFQRIVQEARTMNDFG } \\
M M N Q T N Y S I L P D E D G Y T Q S S Q L L P S D R F I\end{array}$ \\
\hline & 1065 & 1118 & $\begin{array}{l}\text { Q P T P K W R P A Y N I D S D V QTGMLQPNSQWDLVQKFRKQL } \\
\text { SEMFEDPSLQQELGK }\end{array}$ \\
\hline & 1136 & 1183 & $\begin{array}{l}\text { H T D K I Q I VSKLIQGSE SLADTDVNKIFLF HETVITGLNLLS } \\
\text { A I Y V L LN }\end{array}$ \\
\hline & 1192 & 1208 & L D L D T I Q K S I I E W L R E T \\
\hline & 1229 & 1238 & I S E I R N P G L V \\
\hline & 1289 & 1313 & E Q E LA A R Y L V D N Q R I M Q L L L T N IF E \\
\hline & 1323 & 1332 & Q VRFPETSTA \\
\hline & 1334 & 1356 & V H L D F T G L I S L I D S L M A D T K Y F L \\
\hline & 1358 & 1398 & LLRPHIDKN I I Q Y Y EN R SNPGSFYWLEEHLIDKLIKPPTDA \\
\hline & 1400 & 1421 & GRPLPGGELGLEGVNQ I I N K T Y \\
\hline & 1423 & 1433 & L LTKPYNVLQL \\
\hline & 1442 & 1451 & A A N I Q I N N N P \\
\hline & 1476 & 1484 & N S G LR VEQ V \\
\hline & 1486 & 1504 & L G D F R L S N L I R T N N A Q E EN \\
\hline & 1517 & 1536 & YANVNDAANNLRRYRLYGSD \\
\hline & 1541 & 1552 & N N R S M M M V F N Q L \\
\hline & 1559 & 1574 & R F Y D A P S G K I Y L N L I N \\
\hline & 1576 & 1587 & F A N G N F S Q A M E \\
\hline & 1601 & 1620 & A F G H R G D P T E Q S V L L L S L G L \\
\hline & 1622 & 1681 & $\begin{array}{l}\text { LQR L I KDTNRQGLSQHLISTLTEIPIYLKENYRANLPLFNK } \\
M F N \text { I I S Q GELLKQFIQYT }\end{array}$ \\
\hline & 1770 & 1784 & S A M E VLHELTDHPIY \\
\hline & 1786 & 1841 & $\begin{array}{l}\text { ETEEHFIQNYMSRYNKEPLMPFSLSLYYLRDLRIENNEVY } \\
\text { DPLLYPNLESGSPEFK }\end{array}$ \\
\hline & 1843 & 1876 & L Y G T R K L L G N D P V Q L S D M P G V Q L I M K N Y N E T V V A \\
\hline & 1878 & 1889 & E Q ITPTRFEHFY \\
\hline & 1891 & 1899 & HA I O A L R F I \\
\hline & 1901 & 1909 & N I R S F K T V M \\
\hline & 1911 & 1922 & Y N E NTFGG V N L I \\
\hline
\end{tabular}


Table 1: (Continued).

\begin{tabular}{|c|c|c|c|}
\hline ASFV antigen & Start & End & Sequence \\
\hline & 1935 & 1965 & G I G MNAVYSLRKTLQDVISFVESSYQEEQ I N \\
\hline & 1980 & 1997 & L G SN RERER I F N LF D M N I \\
\hline & 1999 & 2039 & PIN VNALMRSIPLANIYNYDYSFEEIACLMYGISAEKVRSL \\
\hline & 2051 & 2059 & V L N I P N R P P \\
\hline & 2061 & 2075 & NTREFMLKLLINPYV \\
\hline & 2077 & 2086 & V S I T Q Y G N E L \\
\hline & 2092 & 2138 & $\begin{array}{l}\text { A G Y M S R I F R D N A L N M GRPKFLSDQ IF N K V LFGSLYPTQF } \\
\text { D Y D E A G P }\end{array}$ \\
\hline & 2140 & 2149 & L A A G I Q R G R E \\
\hline & 2380 & 2397 & E E G P W S I V K Q V G V G I Q K P \\
\hline & 2402 & 2420 & I G K D R F D T R L I R N L I F I T N \\
\hline & 2422 & 2443 & QR L L R LRLNLELSQFR N V L V S P \\
\hline & 2444 & 2476 & H I I N P S I TE Y G F S I T G P SETFS D K Q Y D S D I R I L \\
\hline \multirow[t]{12}{*}{ pp62 } & 1 & 86 & $\begin{array}{l}\text { MPSNMKQFCKISVWLQQHDPDLLEIIN N LCMLGNLSAAK } \\
\text { YKHGVTFIYPKQAKIRDEIKKHAYSNDPSQAIKTLESLILP } \\
\text { FYIPTP }\end{array}$ \\
\hline & 88 & 105 & E F T G E I G S Y T G V K LE V E K \\
\hline & 107 & 120 & E A N K V I L K N G E A V L \\
\hline & 122 & 164 & $\begin{array}{l}\text { PA A D F K P F P R R LA VW I M E S G S P LE GPPYKR KKE G G GN } \\
\text { DP P V }\end{array}$ \\
\hline & 166 & 186 & K H I S P Y T P R T R I A I E V E K A F D \\
\hline & 188 & 253 & $\begin{array}{l}\text { CMRQNWCS VNNPYLAKSVSLLSFLSLNHPTEFIKVLPLID } \\
\text { FDPLVTFYLLLEPYKTHGDDFLIPET }\end{array}$ \\
\hline & 255 & 291 & LFGPTGW NGTDLYQSAMLEFKKFFTQ I TR QTFM D I D \\
\hline & 293 & 312 & ATKEVDVPICYSDPETVHSY \\
\hline & 314 & 324 & N H V R T E I L H H N \\
\hline & 326 & 464 & $\begin{array}{l}\text { VNKVTTPNLVVQAYNELEQTNTIRHYGPIFPESTINALRF } \\
\text { WKKLWRDEQRFVIHGLHRTLMDQPTYETSEFAEIVRNLR } \\
\text { FSRPGNNYINELNITSPAMYGDKHTTGDIAPNDRFAMLVA } \\
\text { FINSTDFLYTAIPEEKVGGN }\end{array}$ \\
\hline & 472 & 504 & T S S L T D L V P T R L H S F L N H L S K LK I L N R A Q Q T V \\
\hline & 506 & 530 & N I L S N D C L N Q L K H Y V K H T G KNEILK \\
\hline \multirow[t]{26}{*}{ p72 } & 25 & 34 & L L N S R I S N I K \\
\hline & 36 & 45 & V N K S Y G K P D P \\
\hline & 58 & 69 & VHF N A H F P Y V P \\
\hline & 71 & 91 & G F E Y N K VRPHTGTPTLGN K L T \\
\hline & 94 & 108 & I P Q Y G D F F H D V V G H \\
\hline & 110 & 124 & L G A C H S S W Q D A P I Q G \\
\hline & 134 & 184 & $\begin{array}{l}\text { LQTFPR N G Y D W D N TPLEGAVYTLVDPFGRPIVPGTKNA } \\
\text { YRNLVYYCEYPG }\end{array}$ \\
\hline & 195 & 210 & VNGNSLDEYSSDVTTL \\
\hline & 212 & 244 & R K F C I P G D K M T G Y K H L V G Q E V S V E G T S G P L L C N \\
\hline & 251 & 268 & P H Q S K P I L T D E N D T QR TC \\
\hline & 270 & 281 & HTNPKFLSOHFP \\
\hline & 286 & 300 & N I Q T A G K Q D I T P I T D \\
\hline & 302 & 310 & TYLD I R R N V \\
\hline & 318 & 331 & QTPKYYQPPLALW I \\
\hline & 340 & 375 & $N \vee N L A$ I P S V S IPFGERFITIKLASQKDLVNEFPGLF \\
\hline & 384 & 402 & G R P S R R N I R F K P W F I P G VI \\
\hline & 410 & 426 & NELYIN N LFVTPE I H N L \\
\hline & 428 & 454 & V K R V R FSLIR VHKTQVTHTNN NHHDEK \\
\hline & 456 & 487 & MSALKWPIEYMFIGLKPTWN ISDQNPHQHRDW \\
\hline & 489 & 500 & KF G H V V N A I M Q P \\
\hline & 507 & 525 & SF Q D R D TALPDACSSISDI \\
\hline & 529 & 555 & TYPITLPIIKN I SVTAHG IN L I D KFPS \\
\hline & 557 & 565 & F C S S Y I PFH \\
\hline & 588 & 611 & PREEYQPSGHIN V SRAREFYISWD \\
\hline & 614 & 626 & Y V G S I T T A D L V V S \\
\hline & 627 & 645 & SA I N F L L Q N G S A V L R Y ST \\
\hline \multirow[t]{6}{*}{ p30 } & 22 & 33 & S S Q V VFHAGSLY \\
\hline & 39 & 55 & E I I N S G R I VT T A I K T L L \\
\hline & 78 & 94 & Q A Q E EW N M I L H V LFEEE \\
\hline & 121 & 136 & ECTSSFETLFEQEPSS \\
\hline & 153 & 165 & V Q H I E Q Y G K A P D F \\
\hline & 191 & 201 & V R L M V I K L LKK \\
\hline \multirow[t]{2}{*}{$\mathrm{CD} 2 \mathrm{v}$} & 296 & 304 & K H V E E I ESP \\
\hline & 322 & 351 & SIHEPSPREPLLPKPYSRYQYNTPIYYMRP \\
\hline
\end{tabular}

$\mathrm{ASFV}=$ African swine fever virus 
Table 2: Highly conserved cytotoxic T-cell epitopes in ASFV.

\begin{tabular}{|c|c|c|c|c|c|c|c|}
\hline ASFV antigen & SLA & Start & End & Epitope & Proteasome score & TAP score & MHC IC $_{50}$ \\
\hline \multirow[t]{24}{*}{ pp220 } & SLA- $1 * 0401$ & 124 & 132 & IADAINQEF & 1.43 & 1.07 & 50.2 \\
\hline & SLA- $1 * 0101$ & 124 & 132 & IADAINQEF & 1.43 & 1.07 & 226 \\
\hline & SLA- $1 * 0101$ & 132 & 140 & FIDLGQDKL & 1.51 & 0.28 & 474.3 \\
\hline & SLA- $1 * 0401$ & 213 & 221 & AVTMIEAVY & 1.43 & 1.44 & 363.9 \\
\hline & SLA- $1 * 0401$ & 344 & 352 & AVNLLRQTF & 1.51 & 1.23 & 276.1 \\
\hline & SLA- $1 * 0401$ & 383 & 391 & RLDRKHILM & 1.05 & 0.15 & 89.1 \\
\hline & SLA- $1 * 0401$ & 393 & 401 & FLNKSTQAY & 1.51 & 1.12 & 69.9 \\
\hline & SLA- $1 * 0801$ & 393 & 401 & FLNKSTQAY & 1.51 & 1.12 & 226 \\
\hline & SLA- $1 * 0401$ & 442 & 450 & ALDLSLIGF & 1.53 & 1.1 & 30.9 \\
\hline & SLA-1*0401 & 501 & 509 & YTDIVQKKY & 1.5 & 1.13 & 53 \\
\hline & SLA-1*0401 & 545 & 553 & AINTFMYYY & 1.38 & 1.33 & 157.3 \\
\hline & SLA-1*0101 & 682 & 690 & FTEATGDTL & 1.66 & 0.28 & 238.6 \\
\hline & SLA- $1 * 0401$ & 788 & 796 & QIYKTLLEY & 1.45 & 1.43 & 73.4 \\
\hline & SLA-1*0801 & 788 & 796 & QIYKTLLEY & 1.45 & 1.43 & 309.4 \\
\hline & SLA- $1 * 0401$ & 1079 & 1087 & SVDVQTGML & 1.38 & 0.36 & 173.4 \\
\hline & SLA-1*0401 & 1362 & 1370 & HIDKNIIQY & 1.48 & 1.15 & 28.9 \\
\hline & SLA- $1 * 0401$ & 1374 & 1382 & RSNPGSFYW & 1.16 & 0.53 & 392.5 \\
\hline & SLA- $1 * 0401$ & 1791 & 1799 & FIQNYMSRY & 1.26 & 1.26 & 180.1 \\
\hline & SLA- $1 * 0401$ & 1832 & 1840 & NLESGSPEF & 1.41 & 1 & 257.4 \\
\hline & SLA- $1 * 0101$ & 1857 & 1865 & LSDMPGVQL & 1.49 & 0.34 & 317.8 \\
\hline & SLA- $1 * 0401$ & 2104 & 2112 & ALNMGRPKF & 1.15 & 1.19 & 238.6 \\
\hline & SLA- $1 * 0401$ & 2125 & 2133 & SLYPTQFDY & 1.02 & 1.37 & 112.5 \\
\hline & SLA- $1 * 0801$ & 2125 & 2133 & SLYPTQFDY & 1.02 & 1.37 & 222.4 \\
\hline & SLA- $1 * 0401$ & 2446 & 2454 & IINPSITEY & 1.33 & 1.28 & 67.3 \\
\hline \multirow{10}{*}{ pp62 } & SLA- $1 * 0401$ & 73 & 81 & TLESLILPF & 1.11 & 1.08 & 202.9 \\
\hline & SLA- $1 * 0101$ & 138 & 146 & IMESGSMPL & 1.01 & 0.39 & 454.2 \\
\hline & SLA- $1 * 0401$ & 227 & 235 & DFDPLVTFY & 1.36 & 1.13 & 324.8 \\
\hline & SLA-1*0401 & 263 & 271 & GTDLYQSAM & 0.78 & 0.03 & 145.1 \\
\hline & SLA- $1 * 0101$ & 263 & 271 & GTDLYQSAM & 0.78 & 0.03 & 256 \\
\hline & SLA-1*0801 & 445 & 453 & FINSTDFLY & 1.2 & 1.31 & 386.2 \\
\hline & SLA- $1 * 0401$ & 445 & 453 & FINSTDFLY & 1.2 & 1.31 & 73.4 \\
\hline & SLA- $1 * 0101$ & 448 & 456 & STDFLYTAI & 0.98 & 0.23 & 98.8 \\
\hline & SLA- $1 * 0401$ & 448 & 456 & STDFLYTAI & 0.98 & 0.23 & 180.1 \\
\hline & SLA-1*0101 & 475 & 483 & LTDLVPTRL & 1.43 & 0.36 & 346.6 \\
\hline \multirow[t]{6}{*}{ p72 } & SLA-1*0401 & 153 & 161 & AVYTLVDPF & 0.83 & 1.29 & 348.4 \\
\hline & SLA-1*0101 & 157 & 165 & LVDPFGRPI & 0.83 & 0.22 & 346.6 \\
\hline & SLA-1*0401 & 199 & 207 & SLDEYSSDV & 0.79 & 0.15 & 373.8 \\
\hline & SLA-1*0401 & 233 & 241 & SVEGTSGPL & 0.95 & 0.46 & 375.9 \\
\hline & SLA-1*0401 & 344 & 352 & AIPSVSIPF & 0.97 & 1.23 & 384.1 \\
\hline & SLA- $1 * 0401$ & 599 & 607 & NVSRAREFY & 1.24 & 1.35 & 258.8 \\
\hline p30 & SLA- $1 * 0401$ & 25 & 33 & VVFHAGSLY & 1.28 & 1.38 & 132.3 \\
\hline $\mathrm{CD} 2 \mathrm{v}$ & SLA- $1 * 0801$ & 340 & 348 & YQYNTPIYY & 1.28 & 1.43 & 245.2 \\
\hline
\end{tabular}

$\mathrm{SLA}=$ Swine leukocyte antigen, $A S F V=$ African swine fever virus

Table 3: Binding energy and binding affinity of SLA-epitope complexes.

\begin{tabular}{lccc}
\hline $\begin{array}{l}\text { Epitope docked } \\
\text { to SLA-01*0401 }\end{array}$ & $\begin{array}{c}\mathbf{\Delta} \mathbf{G}_{\text {bind }} \\
(\mathbf{k J / m o l})\end{array}$ & $\begin{array}{c}\mathbf{K}_{\mathbf{d}} \\
\left(\mathbf{m o l} / \mathbf{d m}^{\mathbf{3}}\right)\end{array}$ & $\begin{array}{c}\text { Antigen } \\
\text { source }\end{array}$ \\
\hline RSNPGSFYW & -46.86 & $1.30 \mathrm{E}-08$ & $\mathrm{pp} 220$ \\
DFDPLVTFY & -46.86 & $1.30 \mathrm{E}-08$ & $\mathrm{p} 62$ \\
AIPSVSIPF & -42.26 & $7.50 \mathrm{E}-08$ & $\mathrm{p} 72$ \\
VVFHAGSLY & -45.61 & $2.10 \mathrm{E}-08$ & $\mathrm{p} 30$ \\
NSDTVGWSW & -51.04 & $2.60 \mathrm{E}-09$ & $\begin{array}{c}\text { Influenza } \\
\text { epitope }\end{array}$ \\
\hline
\end{tabular}

$\mathrm{SLA}=$ Swine leukocyte antigen

negative $\Delta \mathrm{G}_{\text {bind }}$ values with very small $\mathrm{K}_{\mathrm{d}}$. Notice that the $\Delta \mathrm{G}_{\text {bind }}$ and $\mathrm{K}_{\mathrm{d}}$ values of identified cytotoxic T-cell epitopes in this study are comparable to the influenza epitope-SLA complex. Figure-2 shows the plot of sequence position and the RMSD per residue for each complex formed by SLA- $1 * 0401$ with P1, P2, P3, and $\mathrm{P} 4$, and influenza epitopes. All RMSD values in each complex are lower than 0.9 .

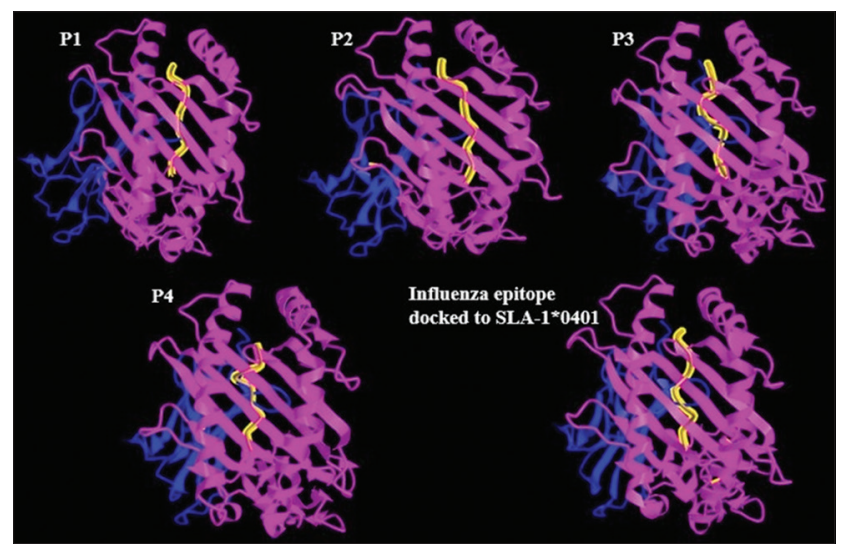

Figure-1: Cytotoxic T-cell epitopes docked to the binding groove of swine leukocyte antigen-1*0401.

\section{Discussion}

The recent emergence of ASFV in new countries and continents has significantly impacted worldwide 


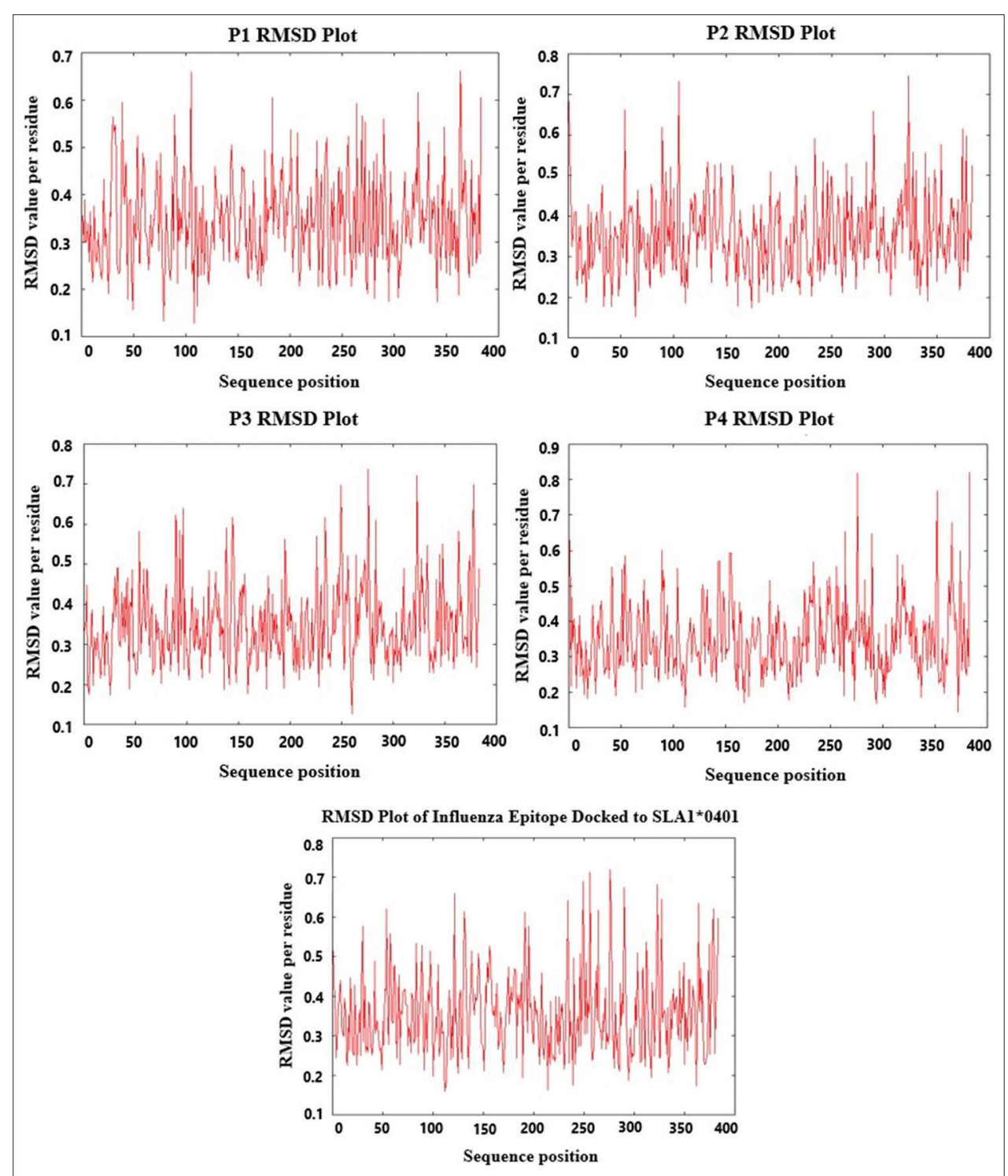

Figure-2: Root-mean-square deviation plots for the residues of swine leukocyte antigen-epitope complexes.

pork production due to its high mortality [27]. At present, there are no commercially available vaccines [28] to treat or prevent domestic swine from this pathogen. Herein, this work aims to identify peptides that can be incorporated as part of a potential vaccine, targeting immunogenic proteins with crucial roles in the virulence of ASFV. Due to higher mutation rates of viral genomic materials, one of the most important properties in vaccines design is the eluding of immune epitope escape by utilizing highly conserved sequences for the identification of epitopes. This strategy may enhance efficacy and longer-term immunity against viral antigens. Therefore, this study utilized highly conserved reference sequences for cytotoxic T-cell epitope mapping. The Shannon variability index of $\leq 0.1$ for all the identified fragments indicates that the reference sequences are highly conserved [29,30]. Cytotoxic T-cell response is critical for effective resolution of viral infections. It has been suggested that the cytotoxic $\mathrm{T}$ lymphocytes have important roles in ASFV protective immunity $[1,31]$. This study identified cytotoxic
T-cell epitopes binding to the widely distributed SLA in pig populations which include SLA- $1 * 0101$, SLA$1 * 0401$, and SLA- $1 * 0801$ [20,32]. All candidate epitopes identified herein, have $\mathrm{MHC} \mathrm{IC}_{50}$ values $\leq 500$ $\mathrm{nmol} / \mathrm{dm}^{3}$ and are classified as good binders [22]. Another significant property that must be considered in any drug and vaccine development procedures is safety. Retrieved epitopes were filtered by conducting in silico toxin-peptide mapping to determine potential toxic epitopes; and Protein BLAST to identify epitopes with significant match to the sequences of Sus scrofa proteome in databases. Short peptides with e-values $<1.0 \mathrm{e}-30$ can be cross-reactive [23]. Candidate epitopes identified in this work can be classified as nontoxic. BLASTp assessments showed that the epitopes have e-values ranging from 0.19 to 75 , making it less likely to cause cell damage and autoimmune reactions in swine. This study identified seven peptides from a total of 26 candidate cytotoxic T-cell epitopes that can promiscuously bind to at least two of the most widely distributed SLAs in swine. Promiscuous epitopes 
include IADAINQEF, FLNKSTQAY, QIYKTLLEY, and SLYPTQFDY in pp220; and GTDLYQSAM, FINSTDFLY, and STDFLYTAI in pp62. These cytotoxic T-cell peptides can potentially cover wider range of swine populations around the world, thereby increasing peptide vaccine immunogenicity. Moreover, resulting values of binding affinity $\left(\mathrm{K}_{\mathrm{d}}\right)$ which are less than 1.0 and the negative binding energy $\left(\Delta \mathrm{G}_{\text {bind }}\right)$ of the candidate epitopes P1, P2, P3, and P4 with SLA$1 * 0401$ (Table-3), indicate favorable complex formation and validate the cytotoxic T-cell epitope mapping conducted in this study. To further demonstrate the immunogenicity of the candidate epitopes, the stability of peptide-SLA complex was evaluated by plotting the RMSD per residue which often represents the mobility of a residue in molecular dynamics simulation. More stable interactions have weaker mobility; thus, lower RMSD value. The RMSD values of all the residues in each peptide-SLA complex formed range from 0.0 to $0.9 \AA$, indicating stable interactions [33]. This is despite the use of the weakest (with the highest $\mathrm{MHC} \mathrm{IC}_{50}$ ) cytotoxic T-cell epitopes (P1, P2, P3, and P4) from pp220, pp62, p72, and p30 in the docking procedures and molecular dynamics evaluations with SLA- ${ }^{*} 0401$. In addition, the value of $\mathrm{K}_{\mathrm{d}}, \Delta \mathrm{G}_{\text {bind }}$, and RMSD plot of influenza A H1N1 virus cytotoxic T-cell epitope bound to the crystal structure of SLA- $1 * 0401$ was also evaluated as a control, to further highlight the positive binding of identified candidate cytotoxic T-cell epitopes to SLA. Table-3 and Figure-2 show that the binding affinity, binding energy, and RMSD plot of influenza A H1N1 epitope are comparable to the values estimated for candidate epitopes predicted in this work.

\section{Limitations of the study}

This study has provided preliminary data for the potential immunotherapeutic targets against ASFV. A computational approach was conducted in the analysis of highly conserved sequences and epitopes mapped from the structural proteins of ASFV. In addition, only cytotoxic T-cell epitopes of ASFV pp220, pp62, p72, p30, and CD2v were covered in this study. $\mathrm{B}$-cell and helper T-cell epitopes can also be analyzed from the identified highly conserved sequences in succeeding studies. As with the step-by-step processes in any vaccine development, it is anticipated that in vitro and in vivo assays will be conducted in the future before application of the candidate epitopes inferred in this study.

\section{Conclusion}

This is the first work to identify highly conserved cytotoxic T-cell epitopes in the structural proteins pp220, pp62, p72, p30, and CD2v which have important roles in the virulence of ASFV. In silico analysis showed that the candidate epitopes can be safely used in vaccine formulations as they are classified non-toxic and are less likely to cross-react with the domestic swine proteome. The peptides can potentially cover wider domestic swine populations and can bind to their corresponding SLA alleles with stability which indicates that the epitopes are potentially immunogenic. Finally, highly conserved cytotoxic T-cell epitopes identified in this study may avoid immune evasion to offer longer and more effective protection against ASFV infection. The use of these candidate epitopes as part of future peptide or recombinant vaccines is anticipated to be validated both in vitro and in vivo in subsequent studies.

\section{Authors' Contributions}

LRMH: Conceived the study. LRMH and EPB: Drafted the manuscript and analyzed the data. Both authors revised the manuscript and approved the final manuscript.

\section{Acknowledgments}

The authors would like to thank the Polytechnic University of the Philippines for their support. The authors did not receive any funds for this study.

\section{Competing Interests}

The authors declare that they have no competing interests.

\section{Publisher's Note}

Veterinary World remains neutral with regard to jurisdictional claims in published institutional affiliation.

\section{References}

1. Takamatsu, H.H., Denyer, M.S., Lacasta, A., Stirling, C.M., Argilaguet, J.M., Netherton, C.L., Oura, C.A., Martins, C. and Rodríguez, F. (2013) Cellular immunity in ASFV responses. Virus Res., 173(1): 110-21.

2. Montgomery, R.E. (1921) On a form of swine fever occurring in British East Africa (Kenya Colony). J. Comp. Pathol. Ther., 34(3): 159-91.

3. Schulz, K., Staubach, C. and Blome, S. (2017) African and classical swine fever: Similarities, differences and epidemiological consequences. Vet. Res., 48(1): 84.

4. Niederwerder, M.C., Stoian, A.M.M., Rowland, R.R.R., Dritz, S.S., Petrovan, V., Constance, L.A., Gebhardt, J.T., Olcha, M., Jones, C.K., Woodworth, J.C., Fang, Y., Liang, J. and Hefley, T.J. (2019) Infectious dose of African swine fever virus when consumed naturally in liquid or feed. Emerg. Infect. Dis., 25(5): 891-897.

5. Cackett, G., Matelska, D., Sýkora, M., Portugal, R., Malecki, M., Bähler, J., Dixon, L. and Werner, F. (2020) The African swine fever virus transcriptome. J. Virol., 94(9): e00119-20.

6. Salas, M.L. and Andrés, G. (2013) African swine fever virus morphogenesis. Virus Res., 173(1): 29-41.

7. Chapman, D.A., Darby, A.C., Da Silva, M., Upton, C., Radford, A.D. and Dixon, L.K. (2011) Genomic analysis of highly virulent Georgia 2007/1 isolate of African swine fever virus. Emerg. Infect. Dis., 17(4): 599-605.

8. Jia, N., Ou, Y., Pejsak, Z., Zhang, Y. and Zhang, J. (2017) Roles of African swine fever virus structural proteins in viral infection. J. Vet. Res., 61(2): 135-143.

9. Andrés, G., Simón-Mateo, C. and Viñuela, E. (1997) Assembly of African swine fever virus: role of polyprotein pp220. J. Virol., 71(3): 2331-2341. 
10. Rubio, D., Alejo, A., Rodríguez, I. and Salas, M.L. (2003) Polyprotein processing protease of African swine fever virus: Purification and biochemical characterization. $J$. Virol., 7(7): 4444-4448.

11. Andrés, G., Alejo, A., Salas, J. and Salas, M.L. (2002) African swine fever virus polyproteins pp220 and pp62 assemble into the core shell. J. Virol., 76(24): 12473-12482.

12. Liu, Q., Ma, B., Qian, N., Zhang, F., Tan, X., Lei, J. and Xiang, Y. (2019) Structure of the African swine fever virus major capsid protein p72. Cell Res., 29(11): 953-955.

13. Afonso, C.L., Alcaraz, C., Brun, A., Sussman, M.D., Onisk, D.V., Escribano, J.M. and Rock, D.L. (1992) Characterization of P30, a highly antigenic membrane and secreted protein of African swine fever virus. Virology, 189(1): 368-373.

14. Hernaez, B., Escribano, J.M. and Alonso, C. (2008) African swine fever virus protein $\mathrm{p} 30$ interaction with heterogeneous nuclear ribonucleoprotein $\mathrm{K}$ (hnRNP-K) during infection. FEBS Lett., 582(23-24): 3275-3280.

15. Petrovan, V., Yuan, F., Li, Y., Shang, P., Murgia, M.V., Misra, S., Rowland, R.R.R. and Fang, Y. (2019) Development and characterization of monoclonal antibodies against p30 protein of African swine fever virus. Virus Res., 269: 197632.

16. Pérez-Núñez, D., García-Urdiales, E., Martínez-Bonet, M., Nogal, M.L., Barroso, S., Revilla, Y. and Madrid, R. (2015) $\mathrm{CD} 2 \mathrm{v}$ Interacts with adaptor protein AP-1 during African swine fever infection. PLoS One, 10(4): e0123714.

17. Dixon, L.K., Abrams, C.C., Bowick, G., Goatley, L.C., KayJackson, P.C., Chapman, D., Liverani, E., Nix, R., Silk,R. and Zhang, F. (2004) African swine fever virus proteins involved in evading host defence systems. Vet. Immunol. Immunopathol., 100(3-4): 117-134.

18. Patronov, A. and Doytchinova, I. (2013) T-cell epitope vaccine design by immunoinformatics. Open Biol., 3(1): 120139.

19. María, R.R., Arturo, C.J., Alicia, J.A., Paulina, M.G. and Gerardo, A.O. (2017) The impact of bioinformatics on vaccine design and development. Vaccines. London: IntechOpen Limited, 123-145

20. Sørensen, M.R., Ilsøe, M., Strube, M.L., Bishop, R., Erbs, G., Hartmann, S.B. and Jungersen, G. (2017) Sequence-based genotyping of expressed swine leukocyte antigen class I alleles by next-generation sequencing reveal novel swine leukocyte antigen Class I haplotypes and alleles in Belgian, Danish, and Kenyan Fattening Pigs and Go"ttingen Minipigs. Front. Immunol., 8: 701.

21. Karosiene, E., Lundegaard, C., Lund, O. and Nielsen, M. (2012) NetMHCcons: A consensus method for the major histocompatibility complex Class I predictions. Immunogenetics, 64(3): 177-186.

22. Jensen, K.K., Andreatta, M., Marcatili, P., Buus, S., Greenbaum, J.A., Yan, Z., Sette, A., Peters, B. and Nielsen, M. (2018) Improved methods for predicting peptide binding affinity to MHC class II molecules. Immunology, 154(3): 394-406.

23. Hileman, R.E., Silvanovich, A., Goodman, R.E., Rice, E.A., Holleschak, G., Astwood, J.D. and Hefle, S.L. (2002) Bioinformatic methods for allergenicity assessment using a comprehensive allergen database. Int. Arch. Allergy Immunol., 128(4): 280-291.

24. Gupta, S., Kapoor, P., Chaudhary, K., Gautam, A., Kumar, R., Open Source Drug Discovery Consortium and Raghava, G.P. (2013) In silico approach for predicting toxicity of peptides and proteins. PLoS One, 8(9): e73957.

25. Xue, L.C., Rodrigues, J.P., Kastritis, P.L., Bonvin, A.M. and Vangone, A. (2016) PRODIGY: A web server for predicting the binding affinity of protein-protein complexes. Bioinformatics, 32(23): 3676-3678.

26. Hospital, A., Andrio, P., Fenollosa, C., Cicin-Sain, D., Orozco, M. and Gelpí, J.L. (2012) MDWeb and MDMoby: An integrated web-based platform for molecular dynamics simulations. Bioinformatics, 28(9): 1278-1279.

27. Woonwong, Y., Do Tien, D. and Thanawongnuwech, R. (2020) The future of the pig industry after the introduction of African swine fever into Asia. Anim. Front., 10(4): 30-37.

28. Sang, H., Miller, G., Lokhandwala, S., Sangewar, N., Waghela, S.D., Bishop, R.P. and Mwangi, W. (2020) Progress toward development of effective and safe African swine fever virus vaccines. Front. Vet. Sci., 7: 84.

29. Shannon, C.E. and Weaver, W. (1997) The Mathematical Theory of Communication. The University of Illinois Press, Urbana.

30. Molero-Abraham, M., Lafuente, E.M., Flower, D.R. and Reche, P.A. (2013) Selection of conserved epitopes from hepatitis C virus for pan-populational stimulation of T-cell responses. Clin. Dev. Immunol., 2013: 601943.

31. Lokhandwala, S., Waghela, S.D., Bray, J., Martin, C.L., Sangewar, N., Charendoff, C., Shetti, R., Ashley, C., Chen, C.H., Berghman, L.R., Mwangi, D., Dominowski, P.J., Foss, D.L., Rai, S., Vora, S., Gabbert, L., Burrage, T.G., Brake, D., Neilan, J. and Mwangi, W. (2016) Induction of robust immune responses in swine by using a cocktail of adenovirus-vectored African swine fever virus antigens. Clin. Vaccin Immunol., 23(11): 888-900.

32. Pedersen, L.E., Harndahl, M., Nielsen, M., Patch, J.R., Jungersen, G., Buus, S. and Golde, W.T. (2013) Identification of peptides from foot-and-mouth disease virus structural proteins bound by Class I swine leukocyte antigen (SLA) alleles, SLA-1*0401 and SLA-2*0401. Anim. Genet., 44(3): 251-258.

33. Fu, Y., Zhao, J. and Chen, Z. (2018) Insights into the molecular mechanisms of protein-ligand interactions by molecular docking and molecular dynamics simulation: A case of oligopeptide binding protein. Comput. Math. Methods Med., 2018: 3502514. 